\title{
Cytogenetics of unfertilized human oocytes
}

\author{
M. Djalali, B. Rosenbusch*, M. Wolf and K. Sterzik* \\ Departments of Clinical Genetics and *Obstetrics and Gynecology, University of Ulm, \\ Federal Republic of Germany
}

\begin{abstract}
Summary. During an in-vitro fertilization programme 150 oocytes from 62 women with a mean age of 31 years (range 24-39) remained unfertilized. Successful chromosome analysis was carried out on 96 oocytes by Q-banding: $59(61.5 \%)$ oocytes bore a normal haploid complement, $8(8.3 \%)$ were diploid and $3(3 \cdot 1 \%)$ tetraploid. In 26 $(27 \cdot 1 \%)$ oocytes aneuploidy was observed; these included $9(9 \cdot 4 \%)$ nullisomic, 5 $(5 \cdot 2 \%)$ double nullisomic, $4(4 \cdot 2 \%)$ triple nullisomic and $2(2 \cdot 1 \%)$ disomic oocytes. The remaining $54(36.0 \%)$ oocytes could not be evaluated. A nearly uniform rate of aneuploidy was found for unfertilized oocytes among different donor age groups.
\end{abstract}

Keywords: chromosome study; human oocyte; in-vitro fertilization; aneuploidy

\section{Introduction}

Cytogenetic investigations on spontaneous abortions and liveborns have indicated that errors during first meiotic division are the most common cause of aneuploidy (Hassold \& Matsuyama, 1979; Hassold et al., 1980; Mikkelsen et al., 1980). Studies on chromosome polymorphism have cleariy shown that in patients with Down's Syndrome the extra chromosome 21 is of maternal origin in about $77 \%$ (Mikkelsen et al., 1980). Similar results are known for malsegregation involving the X chromosome and other numerical abnormalities (Race \& Sanger, 1969; Sanger et al., 1977; Mikkelsen et al., 1980). In facilitating these malsegregation events maternal factors appear more effective than paternal ones (Mikkelsen et al., 1980; Juberg \& Mowrey, 1983).

In-vitro fertilization is now an established method. Treatment of the patient for superovulation provides various numbers of oocytes some of which might not be fertilized during in-vitro procedures. Cytogenetic investigation of such oocytes yields information on the rate of non-disjunction events during maternal meiotic division.

\section{Materials and Methods}

The techniques of stimulation, follicle recovery and oocyte culture have been described previously (Sterzik et al., 1987). We applied three different stimulation protocols, including human menopausal gonadotrophin (hMG), clomiphene and hMG combined with clomiphene. The presence of the first polar body was checked within each oocyte. Chromosome analysis was done on oocytes which were not penetrated by spermatozoa. For chromosome preparation a modification of Tarkowski's method (1966) was used. The oocyte was placed in $1 \%$ sodium citrate at room temperature for $30-40 \mathrm{~min}$. Then the oocyte was picked up with a glass micropipette and transferred to a small dish containing fresh Fixative A (water/acetic acid/ethanol; 5:1:4 by vol.); the oocyte was then transferred to the centre of a slide. A few drops of Fixative B (acetic acid/ethanol; 1:3, v/v) were expelled from a height of $3-5 \mathrm{~cm}$ directly onto each oocyte. The slide was then allowed to dry at $37^{\circ} \mathrm{C}$. The chromosomes were stained with quinacrine mustard (QFQ-banding) and photographed under a fluorescence microscope.

\section{Results}

A total of 150 oocytes from 62 women, aged 24-39 years with a mean of 31 years, remained unfertilized: 28 women provided 1,15 women 2,5 women 3,5 women 4,4 women 5,2 women 6,1 
Table 1. Chromosome complements of unfertilized human oocytes

\begin{tabular}{|c|c|c|c|c|c|c|c|}
\hline \multicolumn{8}{|c|}{ Ploidy } \\
\hline $\mathrm{n}$ & $\mathrm{n}-1$ & $n-2$ & $n-3$ & $\mathrm{n}->3$ & $n+1$ & $2 n$ & $4 n$ \\
\hline $\begin{array}{l}23, ?(35) \\
23, X(24)\end{array}$ & $\begin{array}{l}22,-? \quad(4) \\
22, X,-2(I) \\
22, X,-15(!) \\
22, X,-17(1) \\
22, X,-21(1) \\
22, X,-22(1)\end{array}$ & $\begin{array}{l}21,-? \\
21,-X,-18 \\
21, X,-9,-19(1) \\
21, X,-15,-18(1)\end{array}$ & $20,-?(4)$ & $\begin{array}{l}19,-?(5) \\
11,-?(1)\end{array}$ & $\begin{array}{l}24, X,+21(1) \\
24, X,+22(1)\end{array}$ & $?(8)$ & ?(3) \\
\hline
\end{tabular}

No of oocytes is given in parentheses.

?, not karyotyped.

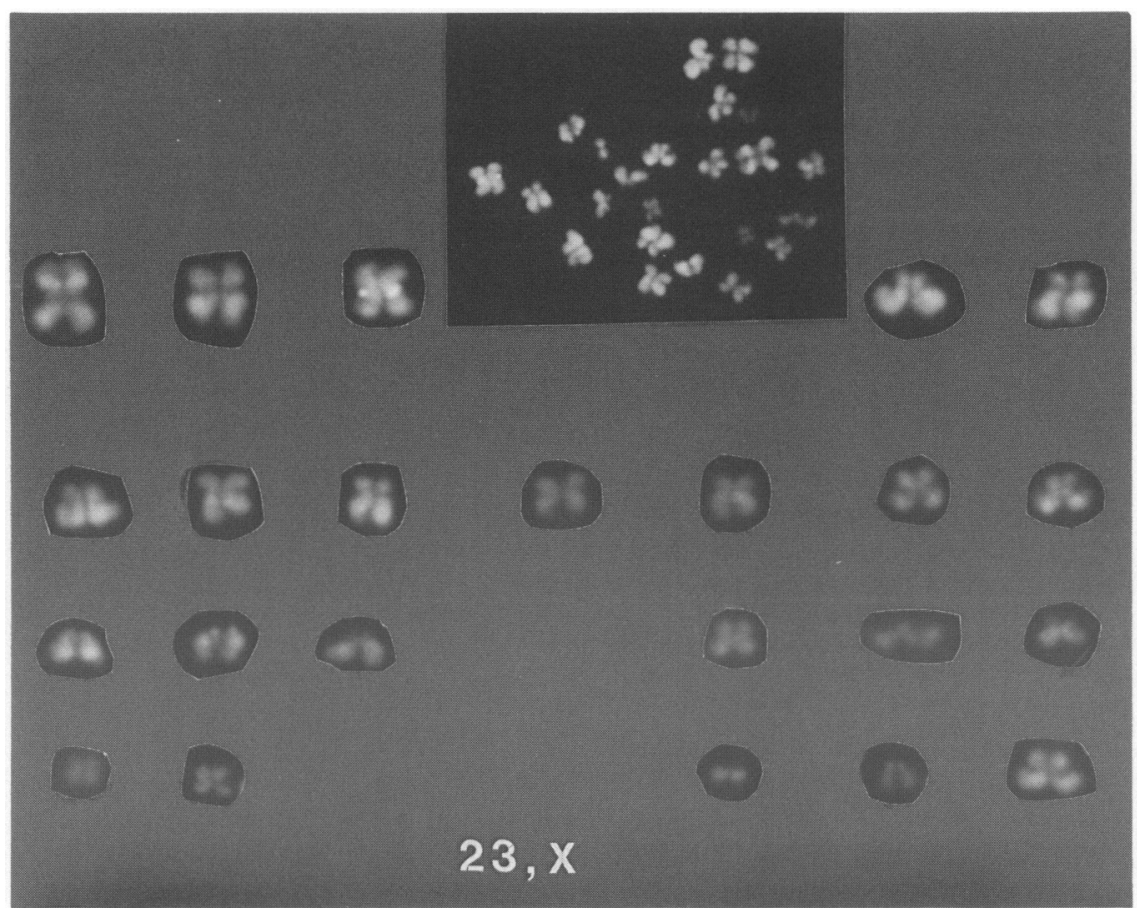

Fig. 1. A karyotype of a 23,X human oocyte demonstrated by QFQ-banding.

woman 7 and 2 women 9 oocytes. There were 54 oocytes $(36.0 \%)$ that could not be analysed because of inferior quality, e.g. chromosome clumping or multiple overlap of chromosomes. The results of the chromosomal evaluation of 96 oocytes are detailed in Tables 1 and 2: $59(61.5 \%)$ oocytes had the normal haploid chromosome complement and 24 of these could be karyotyped by complete banding analysis and revealed normal chromosomes (Fig. 1). Of the oocytes analysed, 37 (38.5\%) were chromosomally abnormal and 10 of these were karyotyped by banding analysis: 8 were diploid and 3 tetraploid. In 26 oocytes aneuploidy was observed, including 9 nullisomic, 


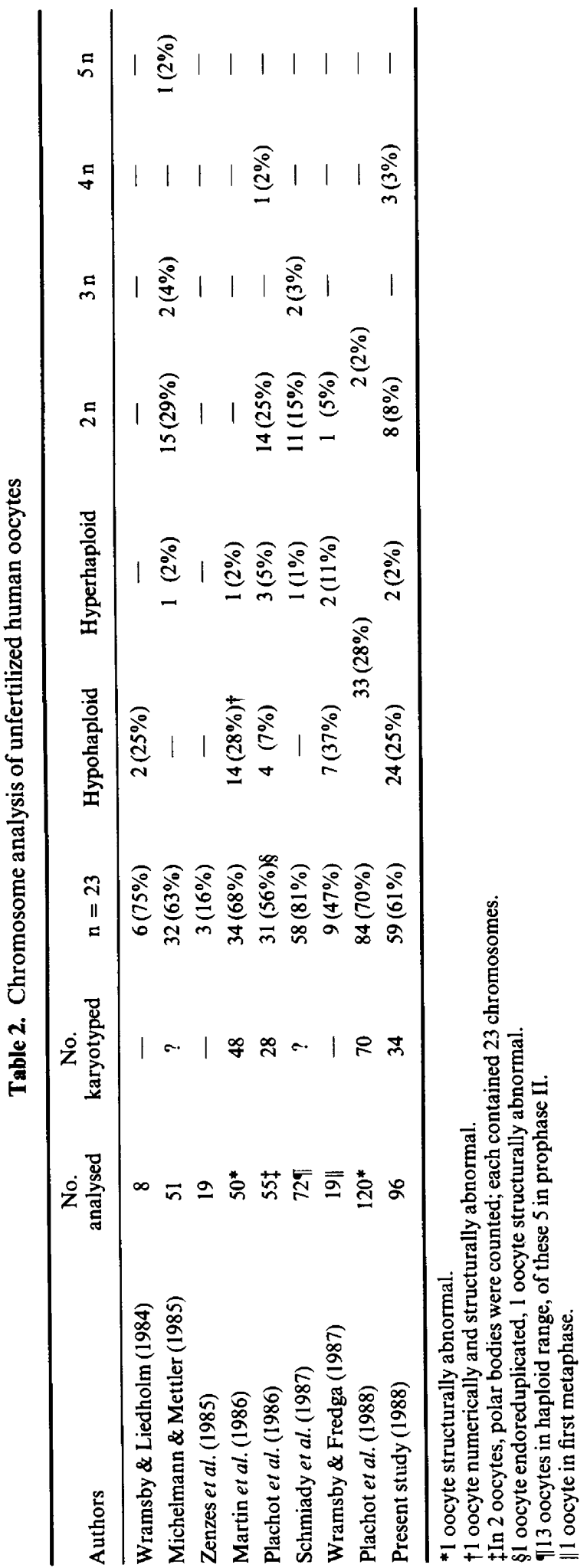




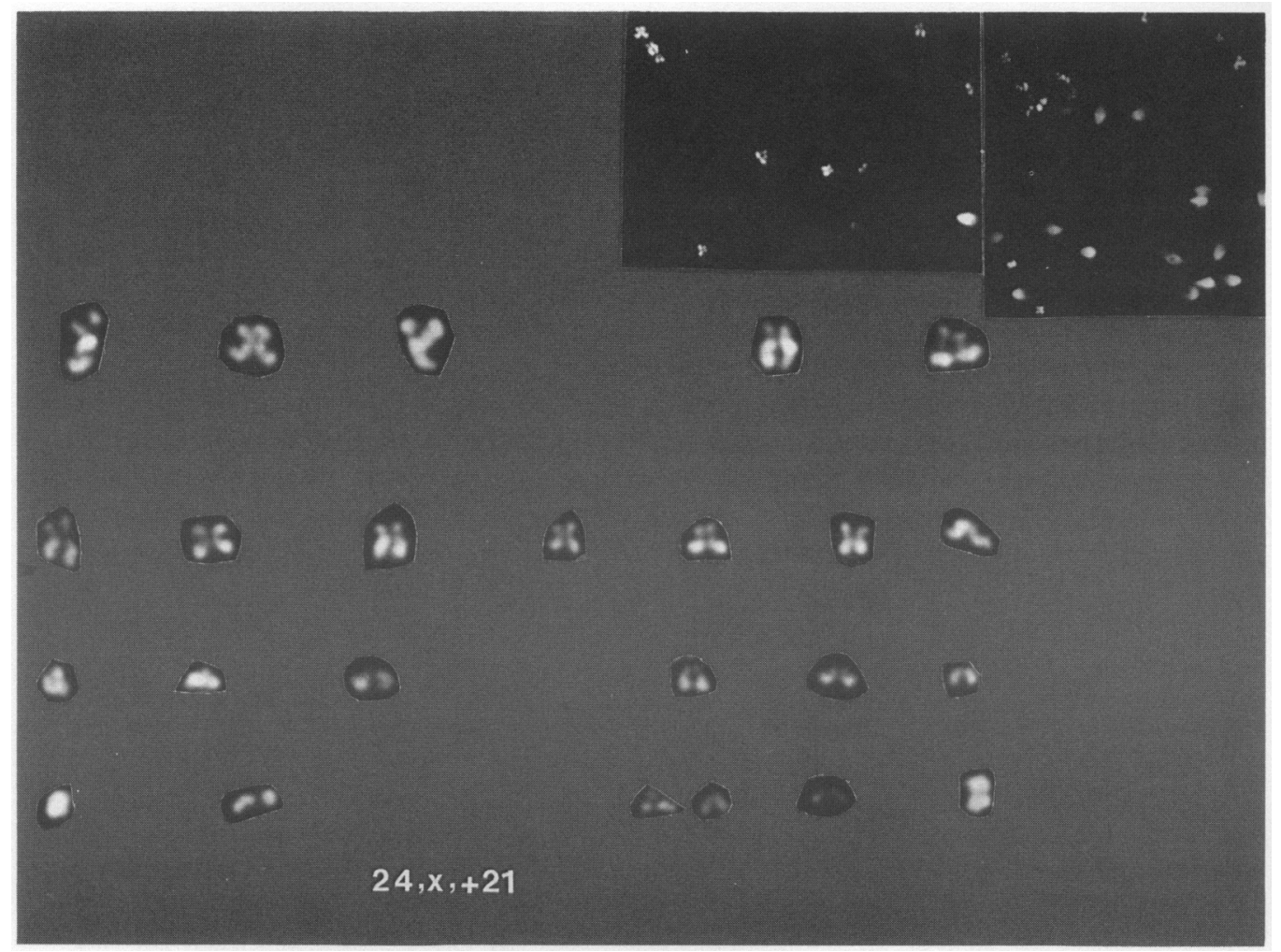

Fig. 2. Karyotype of the $24, \mathrm{X},+21$ human oocyte demonstrated by QFQ-banding.

5 double nullisomic, 4 triple nullisomic and 2 disomic oocytes; the donors of these two disomic oocytes were aged 28 and 33 years (Tables 1 and 2: Figs 2 and 3). No structural aberrations were detected, but some of these might be difficult to diagnose because of limited quality. The overall rate of chromosome aneuploidy in unfertilized oocytes was compared among different donor age groups. The results are shown in Table 3. A nearly constant rate of chromosome aneuploidy was obtained in 3 of the age groups (Table 3 ).

Table 3. Comparison of chromosome aberrations in unfertilized oocytes from women of different ages

\begin{tabular}{|c|c|c|c|c|}
\hline & \multicolumn{4}{|c|}{ Maternal ages (years) } \\
\hline & 2427 & 2831 & 3235 & $36-39$ \\
\hline $\begin{array}{l}\text { Normal } \\
\text { oocytes }\end{array}$ & 6 & 38 & 14 & 1 \\
\hline $\begin{array}{l}\text { Aneuploid } \\
\text { oocytes (\%) }\end{array}$ & $5(45)$ & $18(32)$ & $10(42)$ & 4 \\
\hline
\end{tabular}




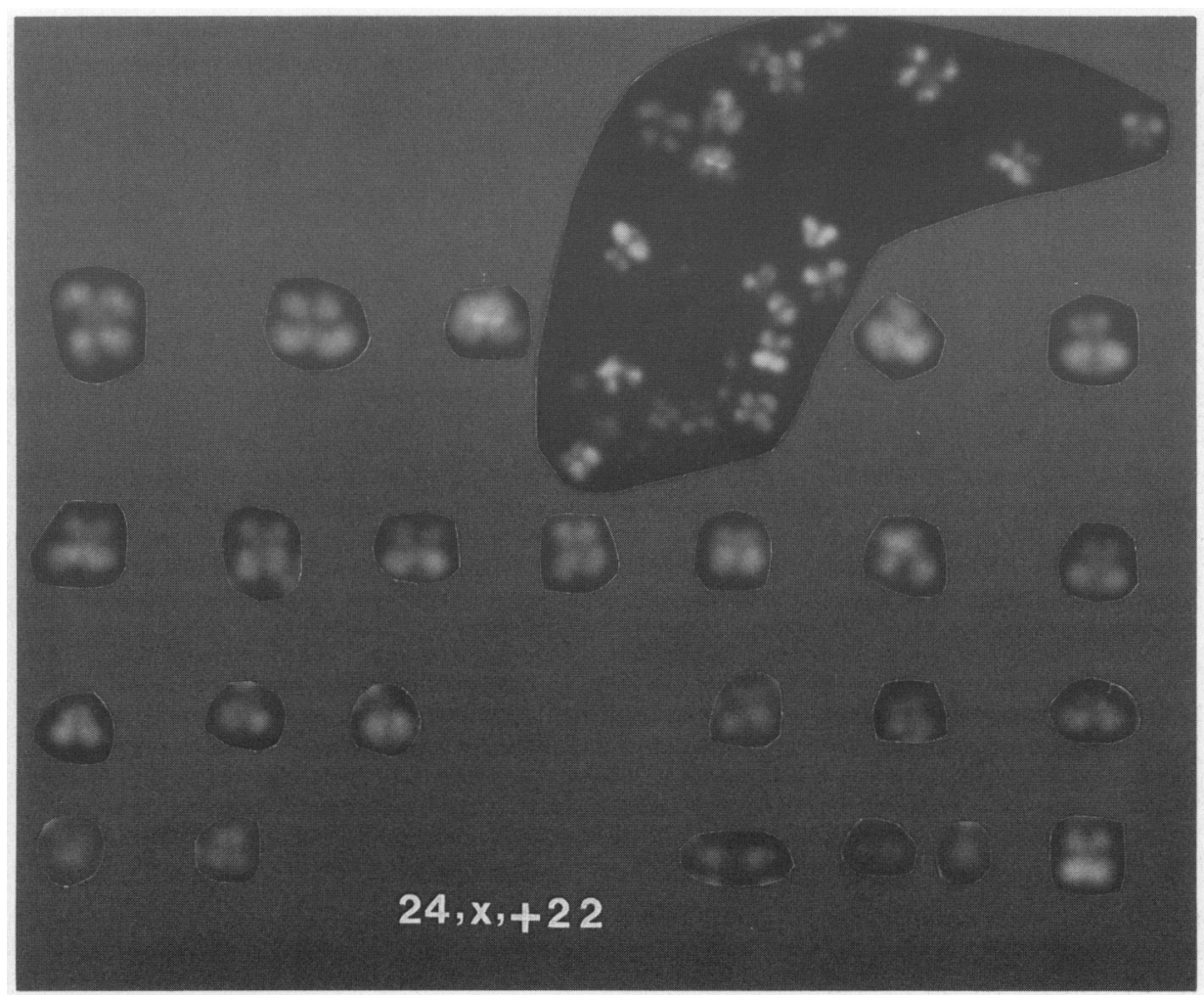

Fig. 3. Karyotype of the $24, X,+22$ human oocyte demonstrated by QFQ-banding.

\section{Discussion}

The high frequency of abnormal chromosome complements obtained in our investigation is not unexpected, since similar findings have been reported (Table 2). Our observation regarding nullisomic, double and triple nullisomic unfertilized oocytes is in agreement with those of Martin et al. (1986) and Plachot et al. (1988) who found similar frequencies of aneuploidy (Table 2). We observed only 2 disomic oocytes, the extra chromosome being in one case a chromosome 21 and in the second a chromosome 22 (Table 1).

Our study on a large series of 96 oocytes allows a correlation between frequency of aneuploidy among different groups of maternal age (Table 3). The number of oocytes analysed in the different age groups was small but no further published data are available. A maternal age effect was not apparent and a nearly uniform rate of aneuploidy within different age groups was observed (Table 3).

The incidence of chromosome aneuploidy within human spermatozoa $(5 \cdot 2 \%)$ has been determined several times (Martin et al., 1983; Martin, 1984): $2 \cdot 4 \%$ and 2.7\% of human spermatozoa are hyper- or hypohaploid, respectively. Our investigation suggests that oocytes with hypohaploid chromosome complements may be more frequent than hyperhaploid ones, although the observed excess of hypohaploidy may be attributed to artificial loss during chromosome preparation (Röhrborn, 1972; Hansmann \& Probeck, 1979; Martin et al., 1986). This observation may support the hypothesis that numerical aberrations in oocytes are due not only to non-disjunction but also to anaphase lagging (Sugawara \& Mikamo, 1980; Mikamo \& Kamiguchi, 1983; Martin et al., [986). 
The incidence of aneuploidy in our study was $27 \cdot 1 \%$ ( $2 \cdot 1 \%$ hyper- and $25 \cdot 0 \%$ hypohaploidy). This overall rate of chromosome aneuploidy is in agreement with other reports (Martin et al., 1986; Veiga et al., 1987; Plachot et al., 1988). Nevertheless, these oocytes were obtained from women with different fertility problems. This may contribute to a higher incidence of chromosome anomalies than in women with normal fertility of similar ages (Plachot et al., 1986, 1988; Wramsby \& Fredga, 1987). More studies with improved cytogenetic techniques are needed to evaluate the accurate rate of aneuploidy in human oocytes.

\section{References}

Hassold, T.J. \& Matsuyama, A. (1979) Origin of trisomies in human spontaneous abortions. Hum. Genet. 46, 285-294.

Hassold, T.J., Jacobs, P., Stein, Z. \& Warburton, D. (1980) Effect of maternal age on autosomal trisomies. Ann. Hum. Gent. 44, 29-36.

Hansmann, I. \& Probeck, H.D. (1979) Detection of nondisjunction in mammals. Environ. Health Perspect. 31, 161-165.

Juberg, R.C. \& Mowrey, P.N. (1983) Origin of nondisjunction in trisomy 21 syndrome: all studies compiled, parental age analysis and international comparisons. Am. J. med. Genet. 16, 111-116.

Martin, R.H. (1984) A comparison of chromosomal abnormalities in hamster egg and human sperm pronuclei. Biol. Reprod. 31, 819-825.

Martin, R.H., Balkan, W., Burns, K., Rademaker, A.W., Lin, C.C. \& Rudd, N.L. (1983) The chromosome constitution of 1000 human spermatozoa. Hum. Genet. 63, 305-309.

Martin, R.H., Mahadevan, M.M., Taylor, P.J., Hildebrand, K., Long-Simson, L., Peterson, D., Yamamoto, J. \& Fleetham, J. (1986) Chromosomal analysis of unfertilized human oocytes. J. Reprod. Fert. 78, 673-678.

Michelmann, H.W. \& Mettler, L. (1985) Cytogenetic investigation on human oocytes and early human embryonic stages. Fert. Steril. 43, 320-322.

Mikamo, K. \& Kamiguchi, Y. (1983) Primary incidence of spontaneous chromosome anomalies and their origin and causal mechanisms in chinese hamster. Mut. Res. 108, 265-278.

Mikkelsen, M., Poulsen, H., Grinsted, J. \& Lange, A. (1980) Nondisjunction in trisomy 21. Study of chromosomal heteromorphisms in 110 families. Ann. Hum. Genet. 44, 17-28.

Plachot, M, Junca, A.M., Mandelbaum, J., de Grouchy, J., Salat Baroux, J. \& Cohen, J. (1986) Chromosome investigations in early life. I. Human oocytes recovered in an IVF programme. Hum. Reprod. 1, 547-551.

Plachot, M., de Grouchy, J., Junca, A.-M., Mandelbaum, J., Salat-Baroux, J. \& Cohen, J. (1988) Chromosome analysis of human oocytes and embryos: does delayed fertilization increase chromosome imbalance? Hum. Reprod. 3, 125-127.
Race, R.R. \& Sanger, R. (1969) Xg and sex-chromosome abnormalities. Br. med. Bull. 25, 99-103.

Röhrborn, G. (1972) Frequencies of spontaneous nondisjunction in metaphase II oocytes of mice. Humangenetik 16, 123-125.

Sanger, R., Tippet, P., Gavin, J., Teesdale, P. \& Daniels, G.L. (1977) Xg groups and sex chromosome abnormalities in people of northern European ancestry: an addendum. J. med. Genet. 14, 210-211.

Schmiady, H., Kentenich, H. \& Stauber, M. (1987) Chromosomenanalyse an menschlichen Eizellen mit Entwicklungsstörungen nach in-vitro-Fertilisation (IVF). Fertilität 3, 39-43.

Sterzik, K., Jonatha, W., Keckstein, G., Rossmanith, W., Traub E. \& Wolf, A. (1987) Ultrasonically guided follicle aspiration for oocyte retrieval in an in vitro fertilisation program: further simplification. Int. J. Gynaecol. Obstet. 25, 309-314.

Sugawara, S. \& Mikamo, K. (1980) An experimental approach to the analysis of mechanisms of meiotic non-disjunction and anaphase lagging in primary oocytes. Cytogenet. Cell Genet. 28, 251-264.

Tarkowski, A.K. (1966) An air drying method for chromosome preparations from mouse eggs. Cytogenetics 5, $394-400$.

Veiga, A., Calderon, G., Santalo, J., Barri, P.N. \& Egozcue, J. (1987) Chromosome studies on oocytes and zygotes from an IVF programme. Hum. Reprod. 2, 425-430.

Wramsby, H. \& Fredga, K. (1987) Chromosome analysis of human oocytes failing to cleave after insemination in vitro. Hum. Reprod. 2, 137-142.

Wramsby, H. \& Liedholm, P. (1984) A gradual fixation method for chromosomal preparations of human oocytes. Fert. Steril. 41, 736-738.

Zenzes, M.T., Belkien, L, Bordt, J., Kan, I., Schneider, H.P.G. \& Nieschlag, E. (1985) Cytologic investigation of human in vitro fertilization failures. Fert. Steril. 43, 883-891. 